\title{
N92-18994
}

\section{GPS INTERFEROMETRIC ATTITUDE AND HEADING DETERMINATION: INITIAL FLIGHT TEST RESULTS}

\author{
Frank van Graas and Michael Braasch \\ Ohio University \\ Athens, Ohio
}

\section{SUMMARY}

Attitude and heading determination using GPS interferometry is a well-understood concept. However, efforts have been concentrated mainly in the development of robust algorithms and applications for low dynamic, rigid platforms (e.g. shipboard). This paper presents results of what is believed by the authors to be the first realtime flight test of a GPS attitude and heading determination system. The system is installed in Ohio University's Douglas DC-3 research aircraft. Signals from four antennas are processed by an Ashtech 3DF 24-channel GPS receiver. Data from the receiver are sent to a microcomputer for storage and further computations. Attitude and heading data are sent to a second computer for display on a software generated artificial horizon. Demonstration of this technique proves its candidacy for augmentation of aircraft state estimation for flight control and navigation as well as for numerous other applications.

\section{INTRODUCTION}

The Global Positioning System (GPS) will soon be capable of providing worldwide positioning and timing services to properly equipped users. In addition, interferometric techniques can be used to obtain realtime, highly accurate attitude and heading information from the GPS measurement data as well. GPS interferometry is a technique based on the measurement of phase differences of the GPS carrier signal observed at two or more antennas. Attitude and heading of a platform can be calculated from measurements with two linearly independent interferometers where each interferometer consists of two separate antennas.

GPS interferometry has been used extensively in precise static and kinematic positioning. Experimental results of static relative positioning using GPS interferometry were first reported in 1982 (ref. 1), showing subcentimeter accuracies for baseline lengths between 8 and 52 meters. Further stationary experiments were presented in 1983 (ref. 2). After that time, implementation issues were emphasized, such as the development of fast initialization algorithms (ref. 3). Realtime low dynamic shipboard tests were performed in 1988 (ref. 4), with reported angular accuracies on the order of several degrees for a 60centimeter baseline. The first non-realtime determination of aircraft pitch and heading using one GPS interferometer was reported in 1989 (ref. 5), revealing pitch and heading uncertainties below 1 milliradian for a 23-meter baseline. Today, sub-milliradian accuracies, 
even for 1-meter baselines, are attainable for rigid platforms using off-the-shelf equipment (ref. 6). In this paper, we present methods and results for realtime pitch, roll, and heading determination of a dynamic, flexible platform.

\section{PRINCIPLES OF ATTITUDE DETERMINATION}

In the formalism of rigid body kinematics, attitude is defined by the rotation transformation which relates a coordinate system fixed in space to a coordinate system fixed in the body (ref. 7). For the purposes of aircraft navigation, the space coordinate system typically is defined to be locally level. The positive $\mathrm{x}$-axis is pointed towards north, the positive $\mathrm{y}$-axis is pointed east and the positive z-axis is pointed down. Finally, since the transformation is concerned with rotation only (and not translation), the origin of the space coordinate system is coincident with the aircraft center of gravity. Similarly, the body (or aircraft) coordinate system also has its origin at the aircraft center of gravity. However, for the aircraft coordinate system, the positive $\mathbf{x}$-axis is directed along the fuselage axis towards the nose, the positive $y$-axis points along the right wing (right according to the pilot), and the positive $z$-axis is directed towards the floor of the aircraft (ref. 8).

The two coordinate systems are related through the antenna locations. Under the rigid body assumption, the antenna locations remain fixed in the aircraft coordinate system. Their locations in this system are determined prior to the flight through standard GPS static surveying techniques. While in flight, the antenna locations in the space coordinate system are determined using GPS interferometry. Thus at any given epoch, the antenna locations in the two coordinate systems are related as follows:

$$
[R]_{s}=[T][R]_{a}
$$

where: $[R]_{s}$ is the matrix containing the locations of the antennas in space coordinates. $[R]_{a}$ is the matrix containing the locations of the antennas in aircraft coordinates. [T] is the rotation transformation matrix.

The transformation is a $3 \times 3$ matrix containing the nine direction cosine elements. It is the product of three rotation matrices each of which specifies a rotation about a certain aircraft coordinate system axis. Thus,

$$
[T]=[\phi][\theta][\psi]
$$

where: $[\Phi]$ represents a rotation about the fuselage axis (roll); $[\theta]$ represents a rotation about the wing axis (pitch); and $[\psi]$ represents a rotation about the vertical axis (heading).

The transformation matrix may now be expressed in terms of the three rotations (also known as the Euler angles): 


$$
[T]=\left(\begin{array}{ccc}
\cos \psi \cos \theta & -\sin \psi \cos \phi+\cos \psi \sin \theta \sin \phi & \sin \psi \sin \phi+\cos \psi \sin \theta \cos \phi \\
\sin \psi \cos \theta & \cos \psi \cos \phi+\sin \psi \sin \theta \sin \phi & -\cos \psi \sin \phi+\sin \psi \sin \theta \cos \phi \\
-\sin \theta & \cos \theta \sin \phi & \cos \theta \cos \phi
\end{array}\right)
$$

Determination of the instantaneous values of the Euler angles proceeds as follows. First, the matrix containing the antenna locations in aircraft coordinates is formed prior to the start of attitude determination. Secondly, realtime GPS interferometric measurements are used to form the matrix containing the antenna locations in the space coordinate system. Solving for the transformation matrix involves postmultiplying the space coordinate matrix by the generalized inverse of the aircraft coordinate matrix:

$$
[T]=[R]_{s}[R]_{a}^{T}\left([R]_{a}[R]_{a}^{T}\right)^{-1}
$$

Finally, the Euler angles can be calculated as follows:

$$
\theta=\sin ^{-1}\left(-T_{31}\right) ; \phi=\sin ^{-1}\left(\frac{T_{32}}{\cos \theta}\right) ; \psi=\sin ^{-1}\left(\frac{T_{21}}{\cos \theta}\right)
$$

The calculations resulting in this Euler angle triad have been presented to provide insight into the attitude determination process. However, although this triad is probably the best understood means of attitude and heading expression, it is far from robust and arguably should not be considered for use in a realtime integrated navigation system (ref. 9). This lack of robustness can be seen most easily by examining equations (3) and (5) for the case of $\theta= \pm 90^{\circ}$. Note that although $\theta$ can be recovered, $\phi$ and $\psi$ are lost. As a result, the Euler angle triad is not an all-attitude parameterization. Although many parameterizations of $[T]$ are possible, quaternions offer conciseness, efficiency, and stability not possible with other parameterizations (refs. 8-9).

Further practical considerations involve the aircraft structure. The derivation given above rests on the assumption that the antenna locations are fixed in the aircraft coordinate system. Error mechanisms are thereby introduced by the non-rigid body behavior of the aircraft. The fuselage by itself is a good rigid body approximation, but inclusion of the wings (with their normal flexing) make the overall aircraft far from being so. An additional consideration is more subtle. The derivation assumes that the location of the aircraft center of gravity is known relative to the antenna positions. Although a nominal location can be used, the true location will change over the course of the flight.

\section{GPS INTERFEROMETRY}

Consider a simplified GPS interferometer, as depicted in figure 1, consisting of two GPS antennas and the necessary electronics to determine the instantaneous phase of a received GPS carrier signal. Usually, the distance between the antennas and a GPS satellite is much 
larger than the baseline length between the two antennas, such that the GPS carrier signal can be treated as a plane wave. Under the assumption that the satellite clock and the receiver clock are synchronized, the phase difference $\Delta \phi$ between simultaneous carrier phase measurements at the two antennas can be written as

$$
\Delta \phi=\phi_{1}-\phi_{2}=\underline{b} \cdot \underline{e}+N \lambda
$$

where $\phi_{1}$ and $\phi_{2}$ are the measured carrier phases in wavelengths for antennas 1 and 2 , respectively; $\underline{b}$ is the three-dimensional baseline vector pointing from antenna 1 to antenna 2 ; $\underline{\hat{\varepsilon}}$ is the unit vector pointing from the interferometer to the satellite; and $\mathrm{N}$ is an integer number accounting for the ambiguity due to the limited length of the carrier wavelength $\lambda$. The phase difference between antennas is also referred to as a Single Difference (SD) (ref. 10). The determination of the unit vector to the satellite requires the approximate coordinates of the interferometer and the satellite coordinates. The latter are calculated from the Navigation Data transmitted by the satellites. A standard GPS navigation solution is used to determine the interferometer coordinates. The baseline solution is not very sensitive to the interferometer coordinates, as was shown by Jurgens (ref. 11), because of the large distance to the satellite with respect to the relatively short baseline length of the interferometer.

If the satellite clock and the receiver clock are not synchronized, each of the phase measurements, $\phi_{1}$ and $\phi_{2}$, contain an unknown satellite clock phase offset and a receiver clock phase offset. If the phase measurements are made simultaneously, then the phase difference $\Delta \phi$ does not contain the unknown satellite clock phase offset. Actually, since the time of transmission for each of the phases transmitted by the satellite will be slightly different by an amount $\Delta t=(\Delta \phi \lambda) / c$, a small offset will be present in the phase difference due to the change in the satellite clock offset during the interval $\Delta t$. This offset can be corrected using the satellite clock coefficients transmitted by the satellite in the Navigation Data.

The unknown receiver clock offset can be eliminated by taking the difference between two independent Single Differences, also referred to as a Double Difference (DD)

$$
D D=\Delta \phi_{1}-\Delta \phi_{2}=\underline{b} \cdot\left(\underline{e}_{1}-\underline{e}_{2}\right)+\left(N_{1}-N_{2}\right) \lambda
$$

Thus a Double Difference is a linear combination of four phase measurements obtained from two satellites at two antennas, as shown in figure 2 . This can be extended to eliminate the unknown integer ambiguity number by differencing two Double Differences at two epochs to obtain a Triple Difference. The Triple Difference is useful for static positioning but only provides the rate of change of the Double Difference for dynamic applications. As such it does not provide an accurate instantaneous solution for the baseline vector.

Assuming that the integer ambiguities are known, then using equation (6), it follows that three independent Double Differences are required to solve for the baseline vector $\underline{b}=$ $\left(\mathrm{b}_{1}, \mathrm{~b}_{2}, \mathrm{~b}_{2}\right)^{\mathrm{T}}$ : 


$$
\left(\begin{array}{l}
D D_{1} \\
D D_{2} \\
D D_{3}
\end{array}\right)=\left(\begin{array}{c}
\left(\underline{e}_{1}-\underline{e}_{2}\right)^{\mathrm{T}} \\
\left(\underline{e}_{1}-\underline{e}_{3}\right)^{\mathrm{T}} \\
\left(\underline{e}_{1}-\underline{e}_{4}\right)^{\mathrm{T}}
\end{array}\right)\left(\begin{array}{l}
b_{1} \\
b_{2} \\
b_{3}
\end{array}\right)
$$

Furthermore, if the norm of the baseline vector, $|\underline{b}|$, is known a priori, then only two Double Differences are needed:

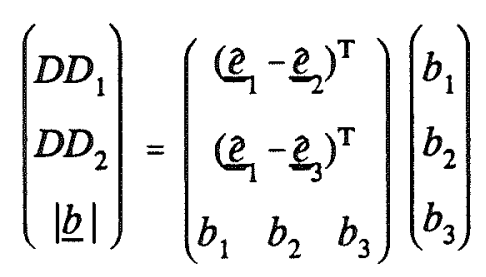

Several techniques have been proposed to quickly determine the integer ambiguity for each of the Double Differences (refs. 12-13). In general, search methods are very effective to quickly resolve the integer ambiguities whenever redundant measurements are available. The basic idea is to evaluate the measurement residuals for potential baseline vector solutions. The techniques used are very similar to those applied to GPS fault detection (see ref. 14 for a summary of these techniques). If, for instance, signals from five satellites are received by one GPS interferometer, four independent Double Differences can be formed. Equation (8) is rewritten as:

$$
\underline{D D}=H \underline{b}
$$

where $\underline{D D}$ is a 4-by-1 Double Difference measurement vector and $\mathrm{H}$ is a 4-by-3 data matrix containing the satellite unit vector differences. The residuals are the differences between the actual Double Difference measurements and those predicted based on the least squares solution for $\underline{b}$, the approximate interferometer coordinates, and the known satellite coordinates. The least squares residual vector, $\underline{e}_{r}$, is obtained by projecting $\underline{D D}$ onto a space perpendicular to the estimation space spanned by the columns of $\mathrm{H}$. Thus, $\underline{e}_{r}$ is orthogonal to the estimation space. An efficient method to obtain $\underline{e}_{r}$ is by decomposing the $H$ matrix into the product of a real orthonormal matrix $\mathrm{Q}$ and an upper triangular matrix $\mathrm{R}$ using the $\mathrm{QR}$ factorization (refs. 15-16). Replacing $\mathrm{H}$ in equation (10) by the product $\mathrm{QR}$ and premultiplying both sides by $\mathrm{Q}^{\mathrm{T}}$ yields $\left(\mathrm{Q}^{\mathrm{T}} \mathrm{Q}=\mathrm{I}\right)$

$$
R \underline{b}=Q^{\mathrm{T}} \underline{D D}
$$

The rank of $\mathrm{R}$ is equal to the rank of $\mathrm{H}$; therefore, the bottom row of $\mathrm{R}$ consists of zeros only. Equation (11) can be divided into two equations: 


$$
\begin{gathered}
U \underline{b}=Q_{1} \underline{D D} \text { or } \underline{b}=U^{-1} Q_{1} \underline{D D} \\
\underline{0}=q \underline{D D}
\end{gathered}
$$

where $U$ consists of the first three rows of $R, Q_{1}$ consists of the first three rows of $Q^{T}$, and $\mathfrak{g}$ is the transpose of the fourth row of $\mathrm{Q}^{\mathrm{T}}$. The top expression of equation (12) relates the measurements to the baseline coordinates, forming the least squares solution. The vector $\mathrm{g}$ and the Double Difference vector $\underline{\mathrm{DD}}$ are othogonal. The latter property is used to obtain the norm of the residual vector if measurement errors are present on the Double Differences:

$$
\underline{e}_{\mathrm{r}} \mid=\left(\begin{array}{llll}
q_{1} & q_{2} & q_{3} & q_{4}
\end{array}\right)\left(\begin{array}{l}
D D_{1} \\
D D_{2} \\
D D_{3} \\
D D_{4}
\end{array}\right)
$$

The vector $\mathrm{g}$ has to be calculated only once for a search at one particular epoch. Thus, to calculate the residual for a potential solution, only four multiplications and three additions are required.

Some prior knowledge is required about the interferometer in order to reduce the potential number of solutions for $\underline{b}$. Several methods can be used, including:

- Limit the baseline length of the interferometer to, for instance, one meter such that the integer ambiguity number must lie in the interval $[-5,5]$ (one GPS wavelength, $\lambda$, is approximately 0.19 meters);

- Use a priori information about the orientation of the interferometer such that the search can be limited to correspond to the approximate baseline orientation. Approximate attitude and heading information could for instance be obtained from a low cost three-axis accelerometer and magnetometer;

- Use Double Differences calculated from the GPS pseudorange measurements (code phase Double Differences) to obtain approximate values for the carrier phase Double Differences.

If the Double Difference uncertainty can be limited to $\pm 5 \lambda$, then even an exhaustive search would only require the calculation of $(11)^{4}=14,641$ residuals, which would require seven times that many or 102,487 floating point calculations. This can easily be accomplished within one second using a microcomputer. In this paper, we estimate the initial DD ambiguities by filtering the relatively noisy code phase Double Differences with the carrier phase Double Differences using a complementary Kalman filter (see also ref. 17). In the complementary filter, the carrier phase DDs are used to provide a reference for the code phase DDs. The performance of the complementary filter is very similar to the Hatch filter 
(ref. 18), but has much lower computational and storage requirements. Although only one DD is estimated, the complementary filter model can easily be extended to include the rate of change of the DD as well. The equations for the (scalar) complementary Kalman filter are summarized below

$$
\begin{gathered}
\underline{\underline{x}}_{\mathrm{k}}^{-}=\underline{\underline{x}}_{\mathrm{k}-1}^{+}+\left(D D_{\mathrm{k}}-D D_{\mathrm{k}-1}\right) \\
p_{\mathrm{k}}^{-}=p_{\mathrm{k}-1}^{+}+q \\
k_{\mathrm{k}}=p_{\mathrm{k}}^{-}\left(p_{\mathrm{k}}^{-}+r\right)^{-1} \\
{\underline{\mathfrak{P}_{\mathrm{k}}^{+}}}^{+}=\underline{\mathfrak{x}}_{\mathrm{k}}^{-}+k_{\mathrm{k}}\left(z_{\mathrm{k}}-\underline{\mathfrak{x}}_{\mathrm{k}}^{-}\right) \\
p_{\mathrm{k}}^{+}=\left(1-k_{\mathrm{k}}\right) p_{\mathrm{k}}^{-}
\end{gathered}
$$

where $R$ denotes the estimated code phase $D D$ with variance $q ; r$ is the variance of the code phase DD measurement $\mathrm{z}$; and $\mathrm{p}$ is the variance of the estimation error. The first two lines in equation (14) propagate the estimated code phase DD and the estimation error variance from time ( $\mathrm{k}-1)$ to time $\mathrm{k}$, followed by the calculation of the Kalman gain (line 3 ), and the update of the estimated code phase DD and the estimation error variance (lines 4 and 5). Figure 3 shows an example of both code phase DDs and carrier phase DDs for a 20-minute section of a flight test. The DDs were obtained from a GPS interferometer on the fuselage of a DC-3 research aircraft during roll maneuvers. The next section provides a detailed description of the aircraft test bed. Note that the code phase DDs and the carrier phase DDs are separated by an unknown integer wavelength ambiguity. Figure 4 shows both the carrier phase DDs and the filtered code phase DDs. As expected, the filtered code phase DDs follow the dynamics very well. Finally, the difference between the carrier phase DDs and the filtered code phase DDs are shown in figure 5. After the filter is initialized with the first code phase DD, it takes typically less than 20 seconds for the carrier phase $D D$ to be within $\pm 5 \lambda$ with respect to the filtered code phase $\mathrm{DD}$. The remaining differences between the filtered code phase DDs and the carrier phase DDs are due to the code phase multipath from the aircraft structure.

The filtered code phase $\mathrm{DD}$ is used to calculate an initial estimate for the absolute carrier phase DD. Next, an exhaustive search is performed to find those combinations of integer ambiguities that will result in low residuals. Depending on the carrier phase measurement noise and the satellite measurement geometry, more than one potential set of ambiguities may be found. Further eliminations of potential sets are achieved by applying known baseline length constraints. For an interferometer mounted on a semi-rigid platform, the baseline length can be determined to within a few millimeters. The knowledge of the norm of the baseline vector is limited by differential antenna phase center migrations, differential multipath and structural flexing of the platform. Next, additional redundant measurement data can be used to resolve the integer ambiguities, again by examining the residuals. Although statistically unlikely, it would still be possible to select the wrong set of 
ambiguities; however, by continuing to examine all measurement residuals throughout the experiment, the resulting inconsistencies in the data would be quickly detected.

Finally, after the integer ambiguities have been determined for each of the Double Differences, they can be used to maintain aircraft attitude and heading for long periods of time until loss-of-lock occurs. It is also important to add that once the integer ambiguities are resolved, redundant measurements are no longer necessary to maintain attitude and heading. Assuming known baseline lengths, a rigid platform would only require continuous lock on the signals from three satellites at three antennas. In this case, to find the approximate interferometer coordinates, another sensor input, such as the baro-altimeter or a stable frequency reference source, is required. If the baselines are subjected to structural flexing, continuous lock on four satellites at three antennas is required.

\section{DESCRIPTION OF THE TESTBED}

The realtime attitude and heading determination testbed consists of three main components: 1) Aircraft, antennas, and cabling; 2) GPS receiver; 3) Data storage, computation, and display units, as depicted in figure 6. Four microstrip antennas have been installed on Ohio University's Douglas DC-3 research aircraft, N7AP. One antenna is located on each wingtip and two antennas are located on top of the fuselage with one near the nose and the other towards the tail (fig. 7). RG-400 cable is used because of its high phase stability and small physical diameter. Phase stability is essential since each of the four cables is approximately 25 meters in length. The cables feed the signal from the antenna preamplifiers to the GPS receiver located in a standard equipment rack installed in the cabin midway between the nose and tail of the aircraft.

The GPS receiver being used is the Ashtech Three-dimensional Direction Finding (3DF) 24-channel receiver (ref. 6). The 3DF is configured as four 6-channel sections with each section receiving signals from a separate antenna. The receiver is packaged in a ruggedized 3-5/8" X 8-1/2" X 8" housing and requires a 12 watt power source. Realtime measurement data at a selectable rate of up to once per second (both raw and processed) are available over two serial ports at data rates up to 115,200 baud.

The storage, computation and display units consist of two microcomputers. A $25 \mathrm{MHz}$ 80386-based computer receives realtime data from the 3DF, performs storage, computation and sends realtime attitude information to a second computer. The second computer is a $12 \mathrm{MHz} 80286$-based PC. It generates an artificial horizon and heading indicator and drives it with the attitude information supplied by the 80386 machine.

Baselines between the antennas were determined using standard GPS static surveying techniques. The lengths of these baselines are given in Table 1. These measurements have also been verified using a steel tape measure and were found to agree to within 5 millimeters. 


\begin{tabular}{||c|c|}
\hline Baseline & Length in meters \\
\hline ant 1 - ant 2 & 7.781 \\
\hline ant 1 - ant 3 & 14.468 \\
\hline ant 1 - ant 4 & 14.466 \\
\hline ant 2 - ant 3 & 14.941 \\
\hline ant 2 - ant 4 & 14.924 \\
\hline ant 3 - ant 4 & 28.194 \\
\hline
\end{tabular}

Table 1. Antenna baseline lengths for Douglas DC-3 test bed.

\section{GROUND and FLIGHT TESTING}

Ground and flight testing of the GPS attitude and heading determination system started in April of 1991. To date, three demonstration flights have been conducted (April 18, May 8 and May 22). Each flight test lasted for approximately 1.5 to 2 hours, and was preceded by a data collection session on the ramp at Ohio University's airport (UNI) in Albany, Ohio. During the first two flights only raw measurement data were collected which were used for the continued development of realtime attitude and heading determination algorithms. During the third flight, long periods of realtime attitude and heading determination were achieved. Moreover, the 3DF GPS receiver maintained continuous lock on at least 3 satellites at 3 antennas during all maneuvers. This is an important result, because it allows for continuous attitude and heading determination. We also found that whenever the receiver would loose lock on a particular satellite due to temporary shielding by the airframe, the receiver would typically re-acquire the signal within a few seconds.

Aircraft flexing was found to be the main reason for discontinuities in the attitude and heading data. The carrier phase DD residuals would increase which caused the algorithm to re-initiate the integer ambiguity search. This could be avoided by allowing larger DD residuals in combination with the use of a higher baseline length uncertainty.

A representative section of the ramp test results collected from the 3DF receiver on May 22,1991 , is shown in figures 8 through 10 . It should be noted that the aircraft was not static during this data collection session; small changes in pitch, roll and heading angles were introduced by wind gusts and by the flight crew moving around inside the aircraft. In addition, some multipath effects can be observed in the data. During this session, five healthy GPS satellites were visible (satellites 2,6,13,14 and 15). The Position Dilution of Precision (PDOP) was approximately equal to 3 throughout the test. The noise level on the roll and heading angles is on the order of $0.1 \mathrm{mrad}$ ( 0.006 degrees). Pitch angle noise is approximately three time larger because of the shorter baseline used for the pitch angle determination. Absolute accuracy results are not available due to the lack of a reference 
for the attitude data which must be aligned with the phase centers of the GPS antennas. However, previous comparisons between the 3DF and kinematic GPS results were found to agree to within $0.1 \mathrm{mrad}$ (ref. 6).

Realtime data collected from the 3DF receiver during two sections of the third flight test conducted in the evening of May 22,1991 are presented next. The noise levels on the pitch, roll and heading angles are difficult to analyze directly, because of the many attitude and heading variations with relatively large magnitudes due to normal flight. Instead, the emphasis of the results presented in this section is on the capability of GPS interferometry to provide realtime, in flight attitude and heading information. Figure 11 shows the ground track of a 27-minute section of the third flight which was conducted in the vicinity of Ohio University. Figures 12 through 14 show the aircraft heading, roll and pitch angles as a function of time. Note that the noise-like behaviour of the angles are all due to actual aircraft dynamics (the period of the angle variations is on the order of 10 to 20 seconds). Approximately 12 minutes after the start of the run, attitude and heading information was lost for approximately 30 seconds. As discussed earlier, this was caused by poor DD residuals which caused the attitude and heading algorithm to re-initiate the integer ambiguity search. Shown in figure 15 is the lock history of the 3DF receiver; even in the presence of signal shielding due to airplane maneuvers, the receiver maintained lock on a minimum of four satellites at 3 antennas.

Figures 16 through 20 show the results for a 13-minute section of the third flight test during which a series of 10 and 30-degree roll maneuvers were performed. Continuous attitude and heading information was available using four or five GPS satellites. During the 30-degree rolls, only one of the five visible satellites was not observed continually by at least three antennas.

\section{SUMMARY, DEVELOPMENTS AND APPLICATIONS}

The feasibility of using realtime GPS interferometric attitude and heading determination for dynamic semi-rigid platforms has been demonstrated. During the course of this research, several issues have been identified which require further study. Among these are continued development of robust fault detection and isolation algorithms for the detection and correction of cycle slips, characterization and mitigation of multipath, and integration with an inertial measurement unit.

GPS interferometry has numerous applications including: reliable aircraft/spacecraft state estimation; measurement of structural flexing of large space structures; synthetic aperture radar (ref. 5); aerial photogrammetry; pointing systems; and rapid alignment of inertial navigation systems for submarines and aircraft while maneuvering. 


\section{ACKNOWLEDGEMENTS}

This work was supported by the FAA/NASA Joint University Program for Air Transportation Research (Grant NGR-36-009-17). The 3DF GPS receiver was provided by Ashtech, Inc. The authors are indebted to Dr. Javad Ashjaee and Mr. Mark Kuhl for their support, including many technical discussions on the topic of GPS interferometry, and their help in processing the raw measurement data. The authors would also like to thank the DC-3 flight crew, Dr. Richard H. McFarland, Mr. Dennis J. Atkeson and Mr. Richard Zoulek, for their flexibility in dealing with the GPS satellite visibility windows.

\section{REFERENCES}

1. Greenspan, R. L., Ng, A. Y., Przyjemski, J. M., and Veale, J. D.: Accuracy of Relative Positioning by Interferometry with Reconstructed Carrier GPS: Experimental Results. 3rd International Geodetic Symposium on Satellite Doppler Positioning, Las Cruces, NM, Feb. 8-12, 1982.

2. Burgett, W. S., Roemerman, S. D., and Ward, P. W.: The Development and Applications of GPS-Determined Attitude. Presented to the National Telesystems Conference, November 1983.

3. Remondi, B. W.: Performing Centimeter-Level Surveys in Seconds with GPS Carrier Phase: Initial Results. Navigation: Journal of the Institute of Navigation, Vol. 32, No. 4, Winter 1985-86.

4. Kruczynski, L. R., Li, P. C., Evans, A. G., and Hermann, B. R.: Using GPS to Determine Vehicle Attitude: USS Yorktown Test Results. Proceedings of the 2nd International Technical Meeting of the Satellite Division of the Institute of Navigation, Colorado Springs, CO, Sep. 27-29, 1989.

5. Purcell, Jr., G. H., Srinivasan, J. M., Young, L. E., DiNardo, S. J., Hushbeck, Jr., E. L., Meehan, T.K., Munson, T. N. and Yunck, T. P.: Measurement of Aircraft Position, Velocity, and Attitude using Rogue GPS Receivers. Proceedings of the 5th International Geodetic Symposium on Satellite Positioning, Las Cruces, NM, March 1989.

6. Ferguson, K., Kosmalska, J., Kuhl, M., Eichner, J. M., Kepski, K, and Abtahi, R.: ThreeDimensional Attitude Determination with the Ashtech 3DF 24-Channel GPS Measurement System. Proceedings of the National Technical Meeting of the Institute of Navigation, Phoenix, AZ, Jan. 1991.

7. Goldstein, H.: Classical Mechanics, Second edition. Addison-Wesley, Reading, Massachusetts, 1980.

8. Farrell, J. L.: Integrated Aircraft Navigation. Academic Press, San Diego, 1976. 
9. Farrell, J. L. and Van Graas, F.: That All-Important Interface. Proceedings of the Third International Technical Meeting of the Satellite Division of the Institute of Navigation, Colorado Springs, CO, September 1990.

10. Remondi, B. W.: Using the Global Positioning System (GPS) phase observable for relative geodesy: modeling, processing, and results. Ph.D. Dissertation, The University of Texas at Austin, Austin, Texas, 1984.

11. Jurgens, R. D.: Realtime GPS Azimuth Determining System. Proceedings of the National Technical Meeting of The Institute of Navigation, San Diego, CA, Jan. 23-25, 1990.

12. Hatch, R.: Ambiguity Resolution in the Fast Lane. Proceedings of the 2nd International Technical Meeting of the Satellite Division of The Institute of Navigation, Colorado Springs, CO, Sep. 27-29, 1989.

13. Hwang, P. Y. C.: Kinematic GPS: Resolving Integer Ambiguities on the Fly. Proceedings of the IEEE Position, Location, and Navigation Symposium, Las Vegas, NV, March 21-23, 1990.

14. Brown, R. G., Chin, G. Y., and Kraemer, J. H.: RAIM: Will it Meet the RTCA GPS Minimum Operational Performance Standards? Proceedings of the National Technical Meeting of The Institute of Navigation, Phoenix, AZ, Jan. 22-24, 1991.

15. Brenner, M.: Implementation of a RAIM Monitor in a GPS Receiver and an Integrated GPS/IRS. Proceedings of the 3rd International Technical Meeting of the Satellite Division of The Institute of Navigation, Colorado Springs, CO, Sept. 1990.

16. Golub, G. H. and Van Loan, C. F.: Matrix Computations, Second Edition. The Johns Hopkins University Press, Baltimore, MD, 1989.

17. Hwang, P. Y. C. and Brown, R. G.: GPS Navigation: Combining Pseudorange with Continuous Carrier Phase using a Kalman Filter. Proceedings of the 2nd International Technical Meeting of the Satellite Division of The Institute of Navigation, Colorado Springs, CO, Sep. 27-29, 1989.

18. Hatch, R.: The Synergism of GPS Code and Carrier Measurements. Proceedings of the 3rd International Geodetic Symposium on Satellite Doppler Positioning, Las Cruces, NM, Feb. 1982. 


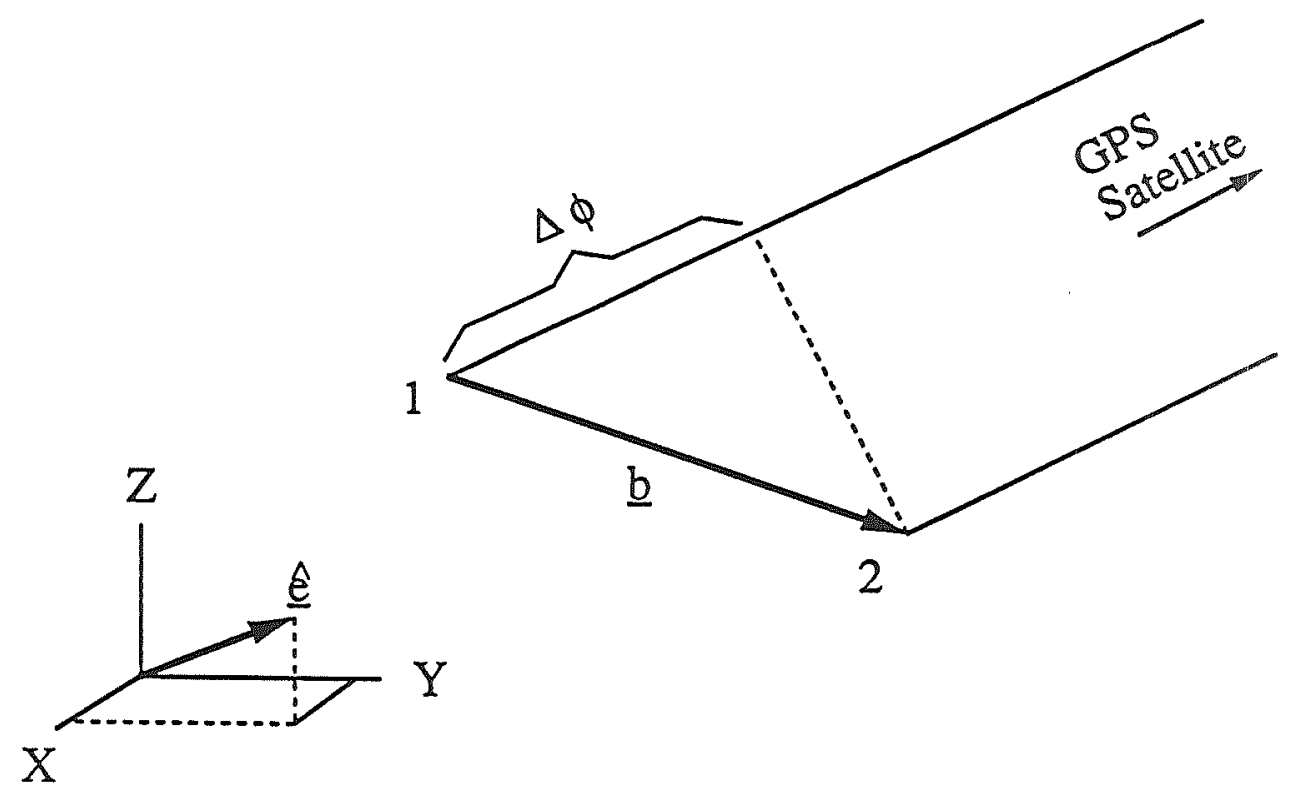

Figure 1. Simplified GPS Interferometer.

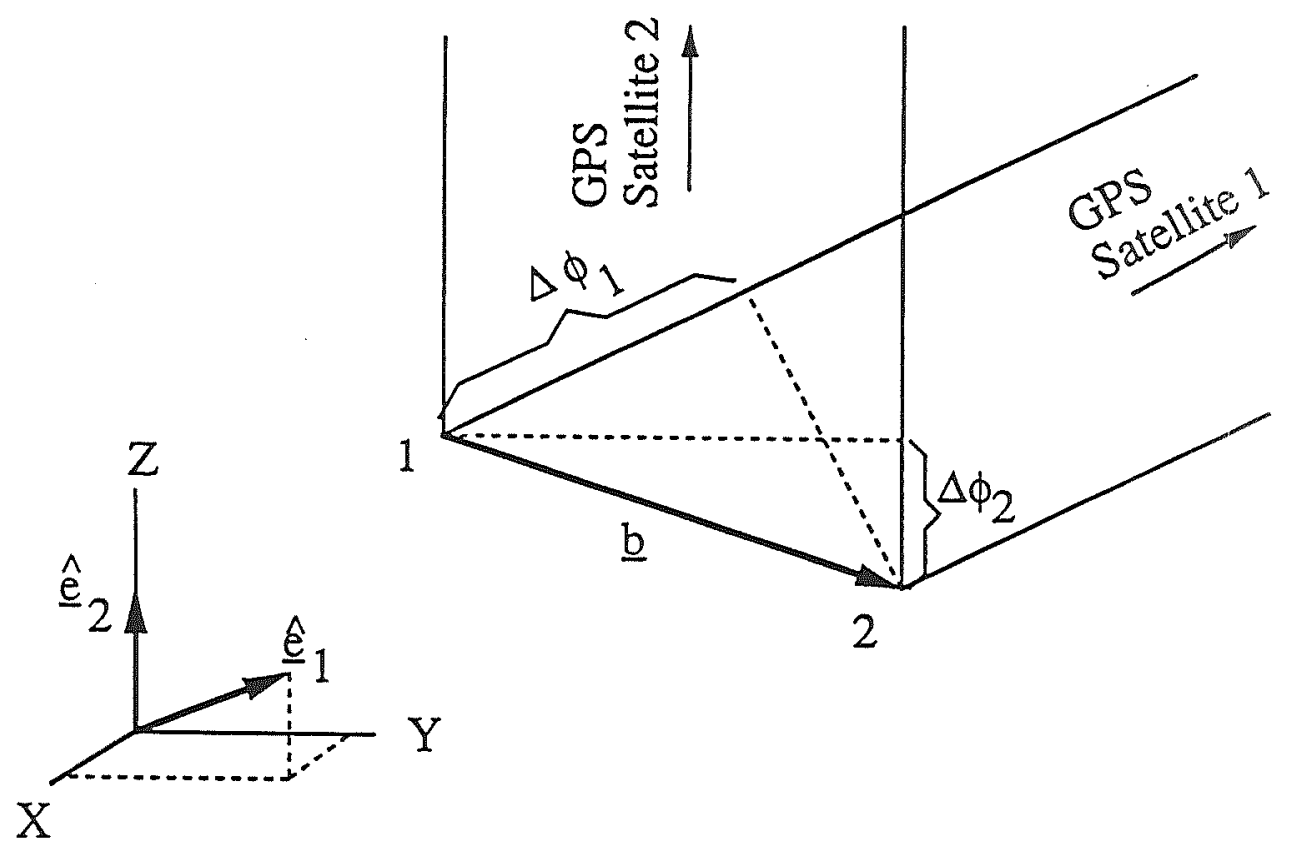

Figure 2. The Double Difference Observation. 


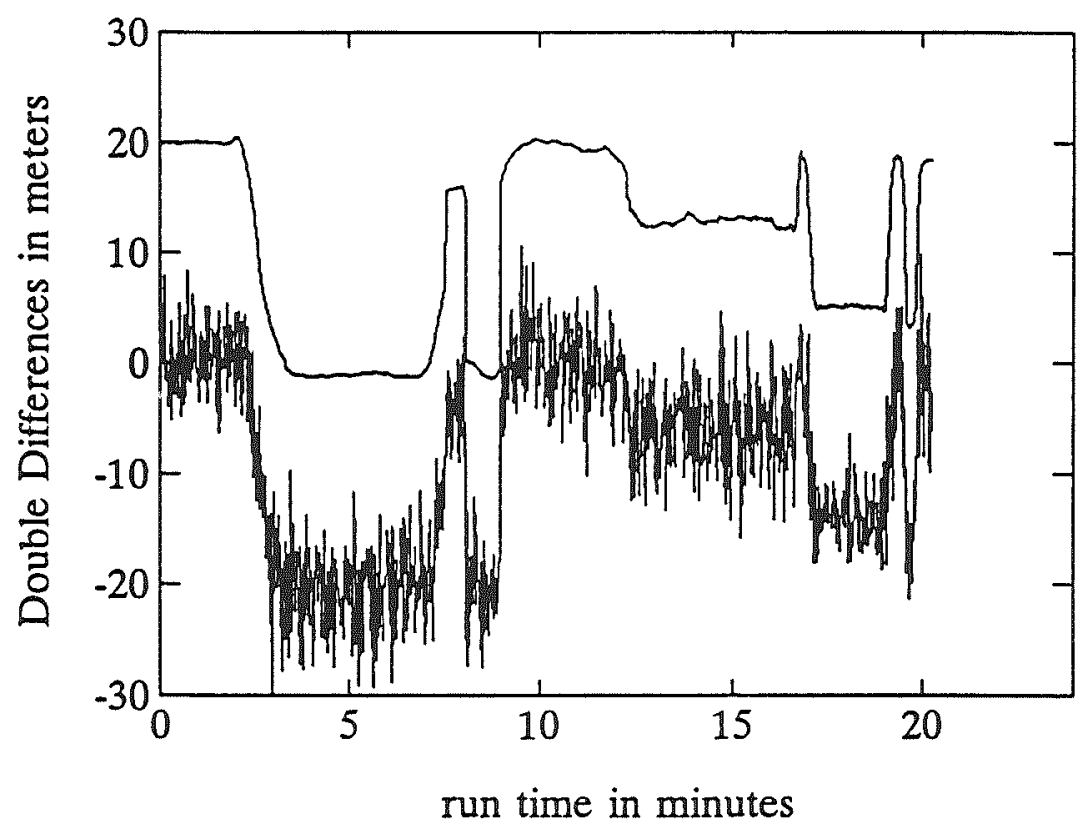

Figure 3. Code phase (bottom) and carrier phase (top) Double Differences.

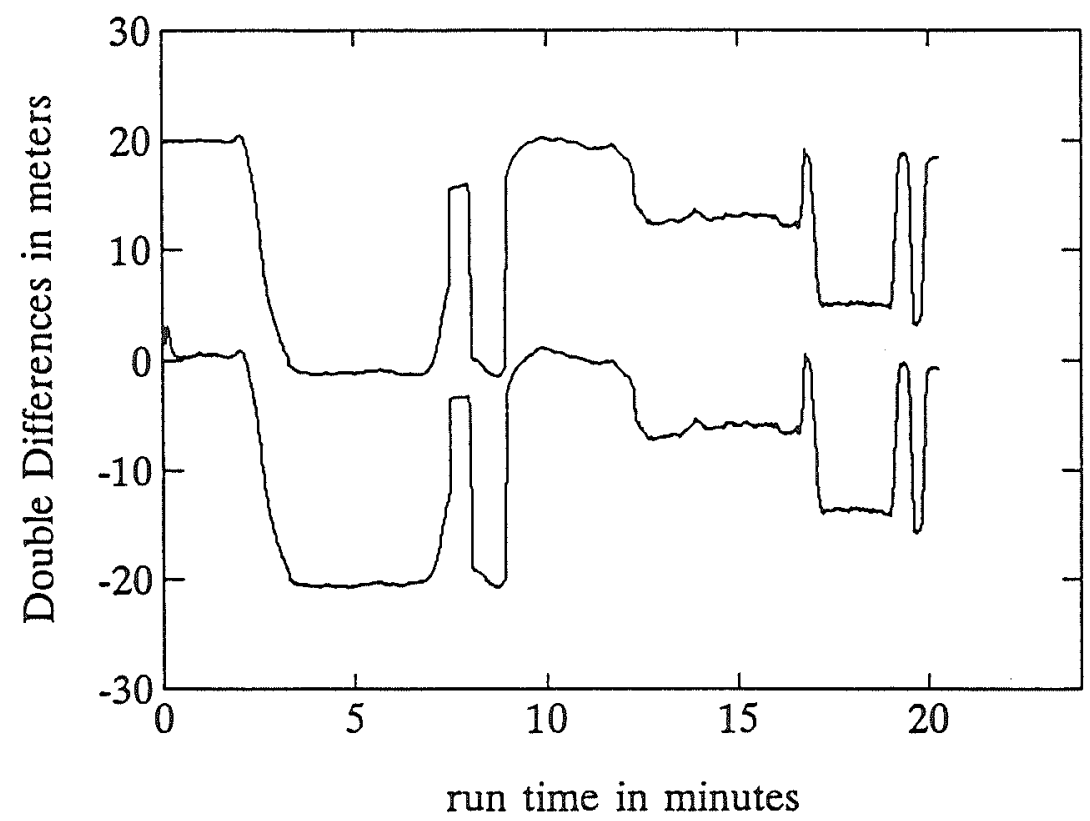

Figure 4. Carrier phase Double Differences (top) and filtered code phase Double Differences (bottom). 


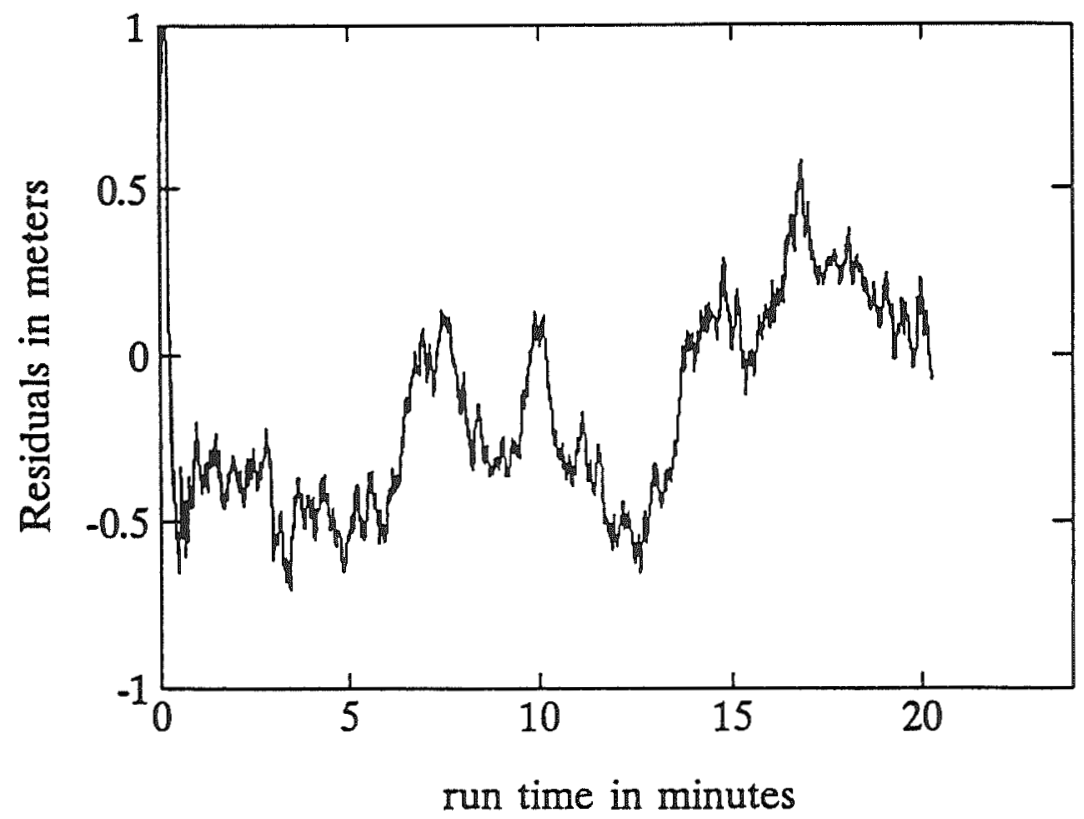

Figure 5. Difference between carrier phase Double Differences and filtered code phase Double Differences.

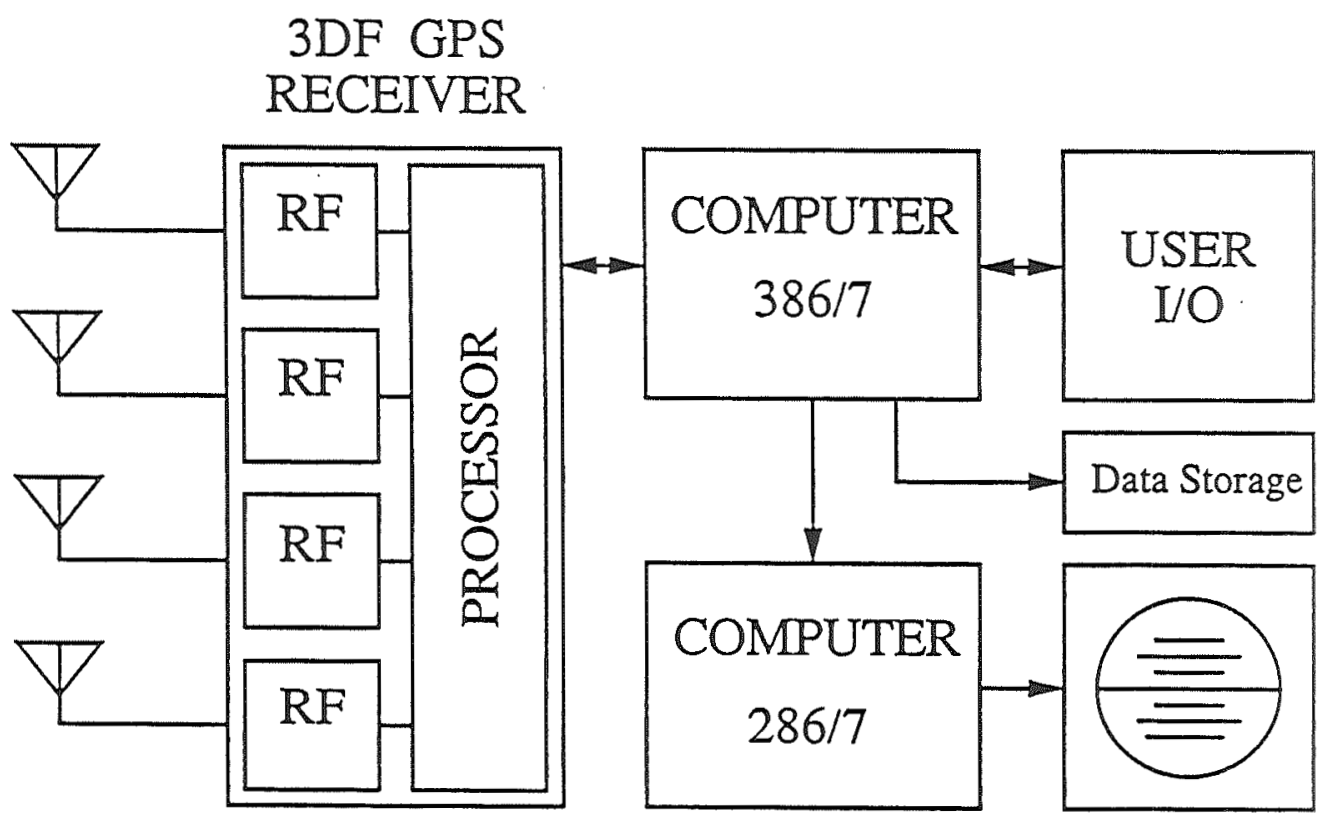

Figure 6. Block diagram of realtime attitude and heading determination test bed. 


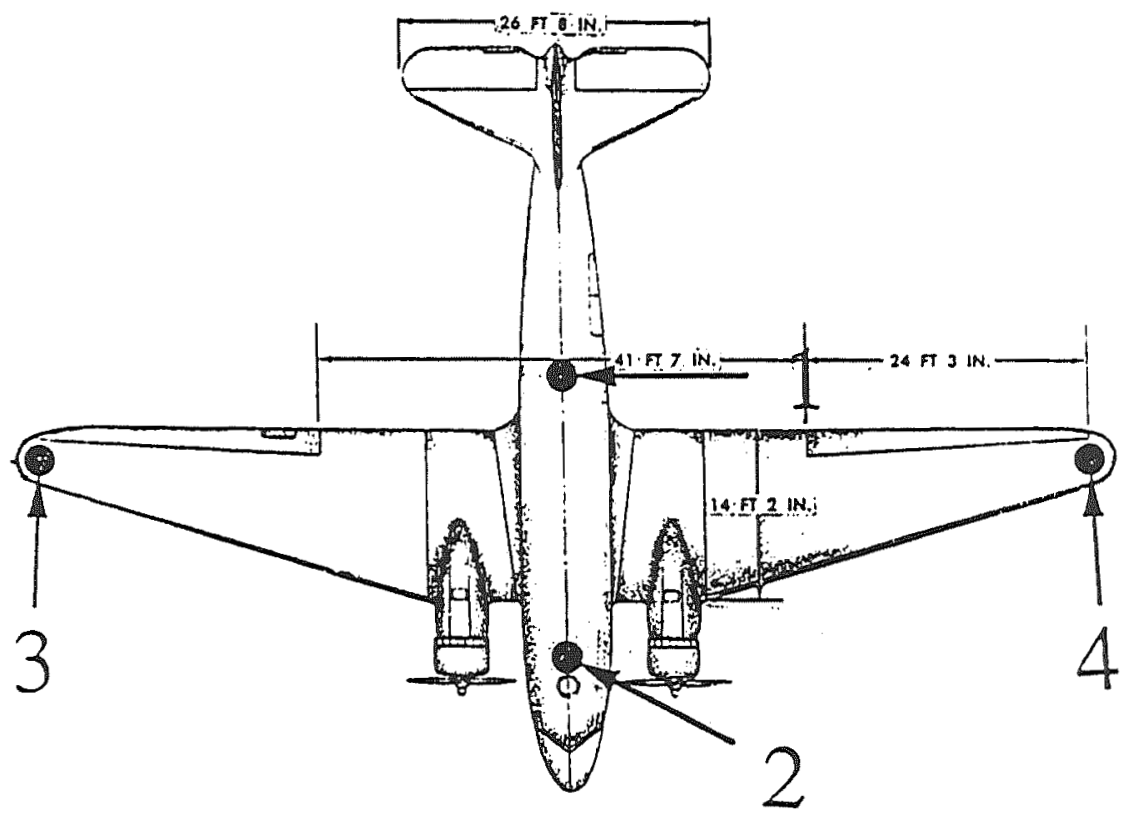

Figure 7. Douglas DC-3 GPS antenna locations.

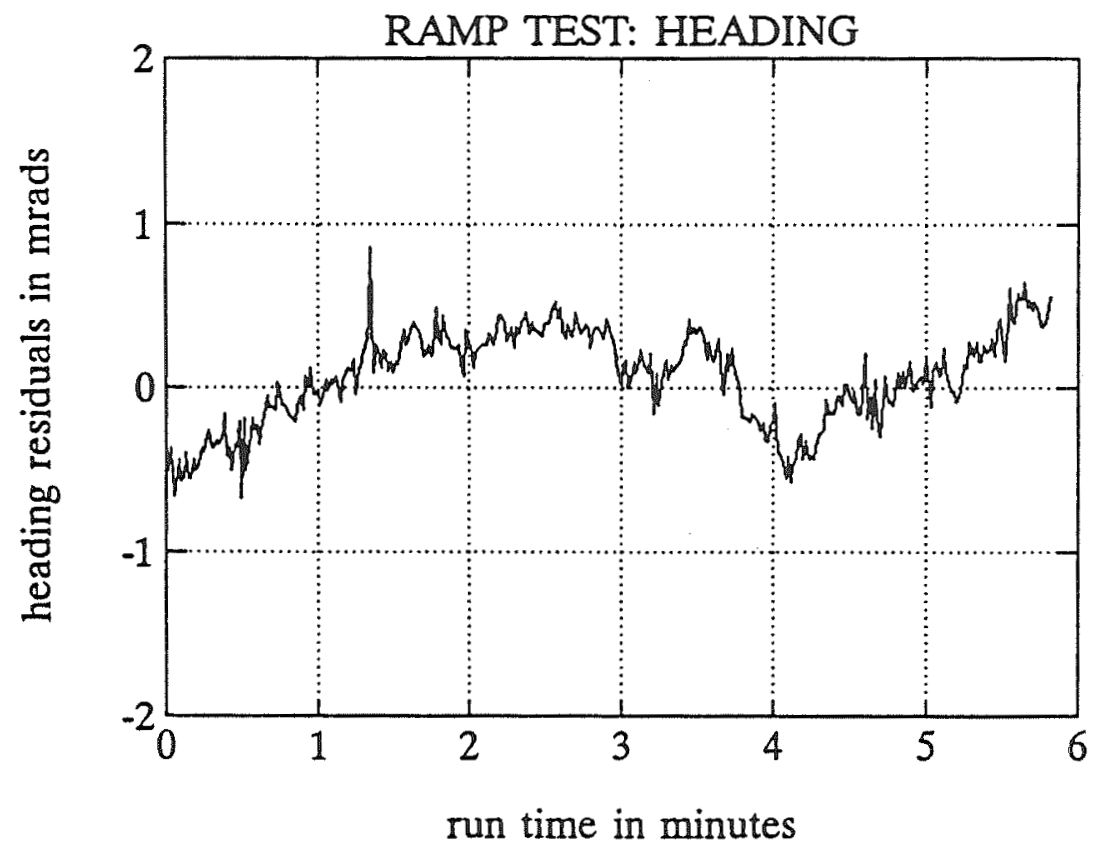

Figure 8. Ramp test heading residuals. 


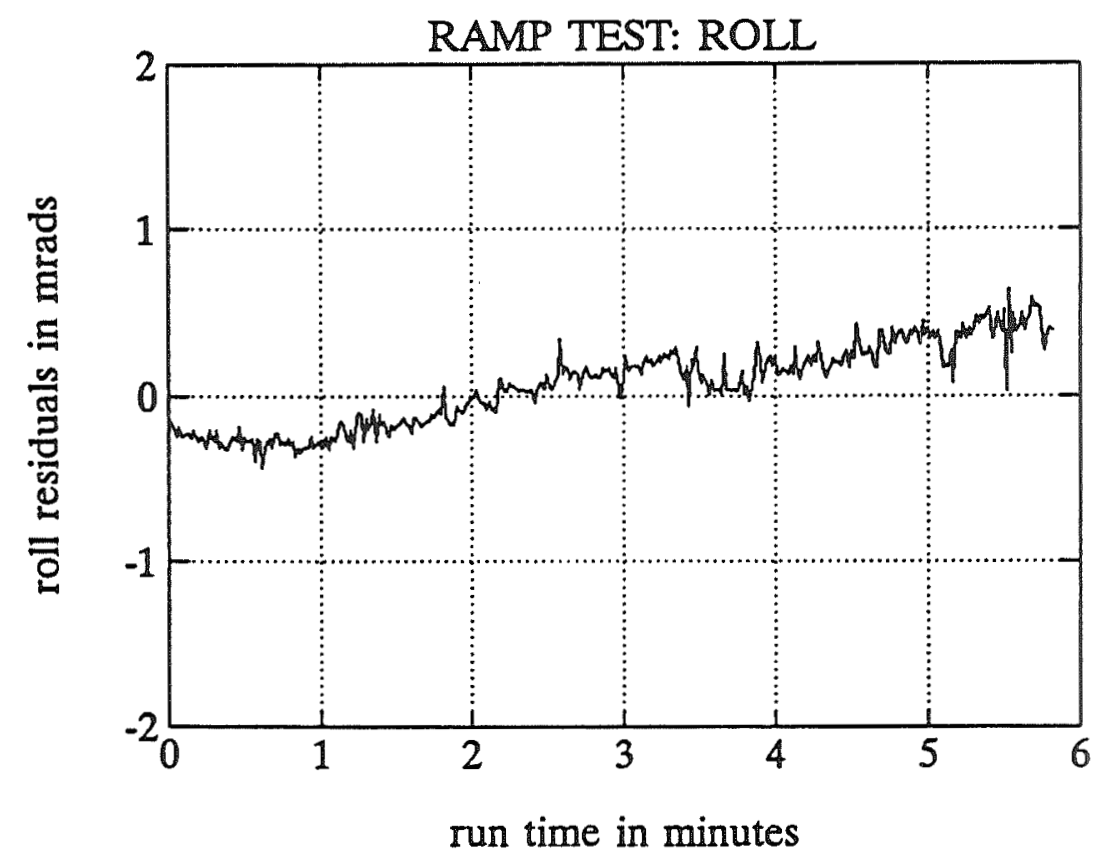

Figure 9. Ramp test roll residuals.

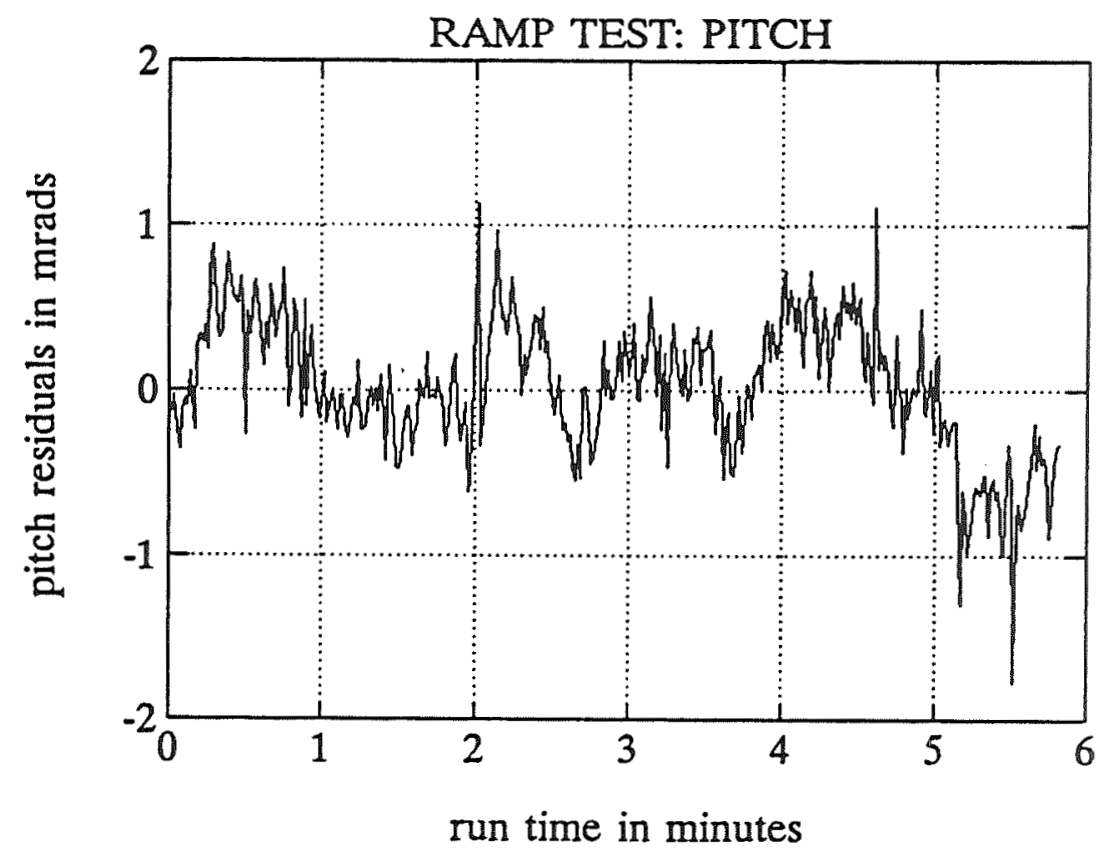

Figure 10. Ramp test pitch residuals. 


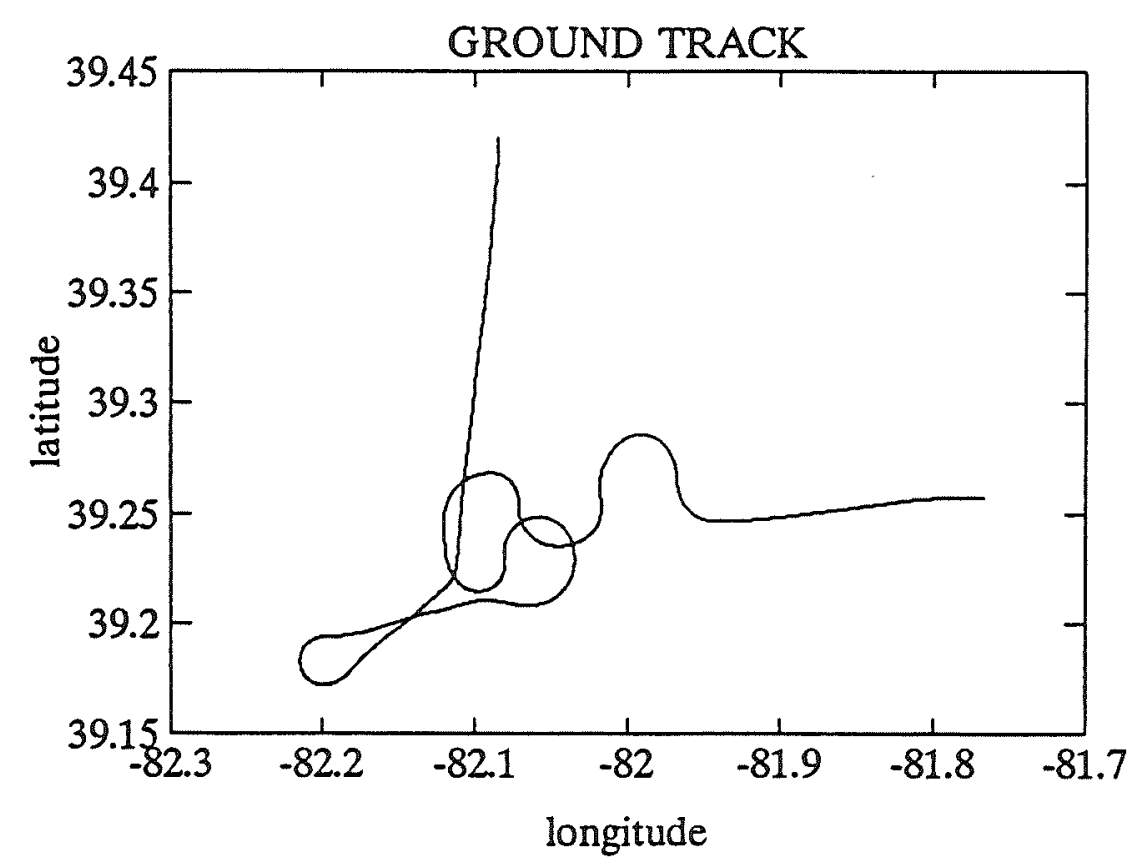

Figure 11. Ground track for a 27 -minute section of the third flight test.

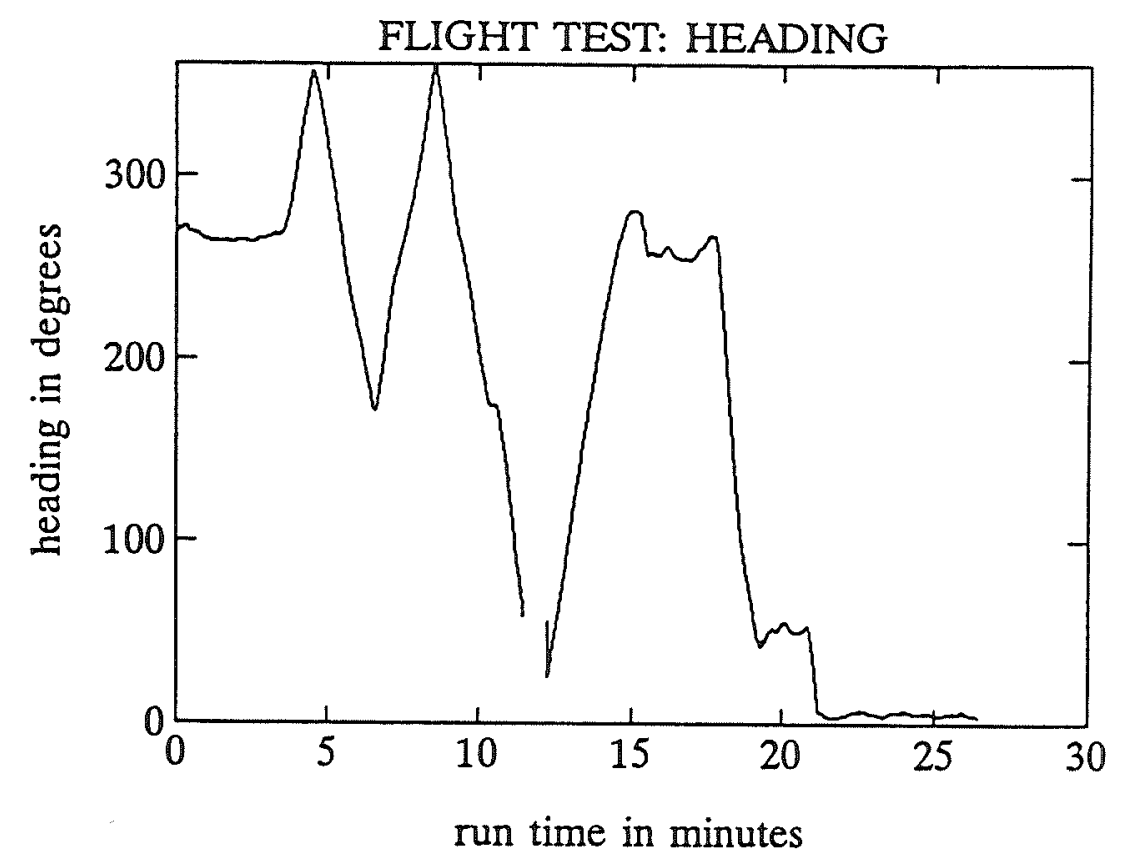

Figure 12. Flight test heading as a function of time. 


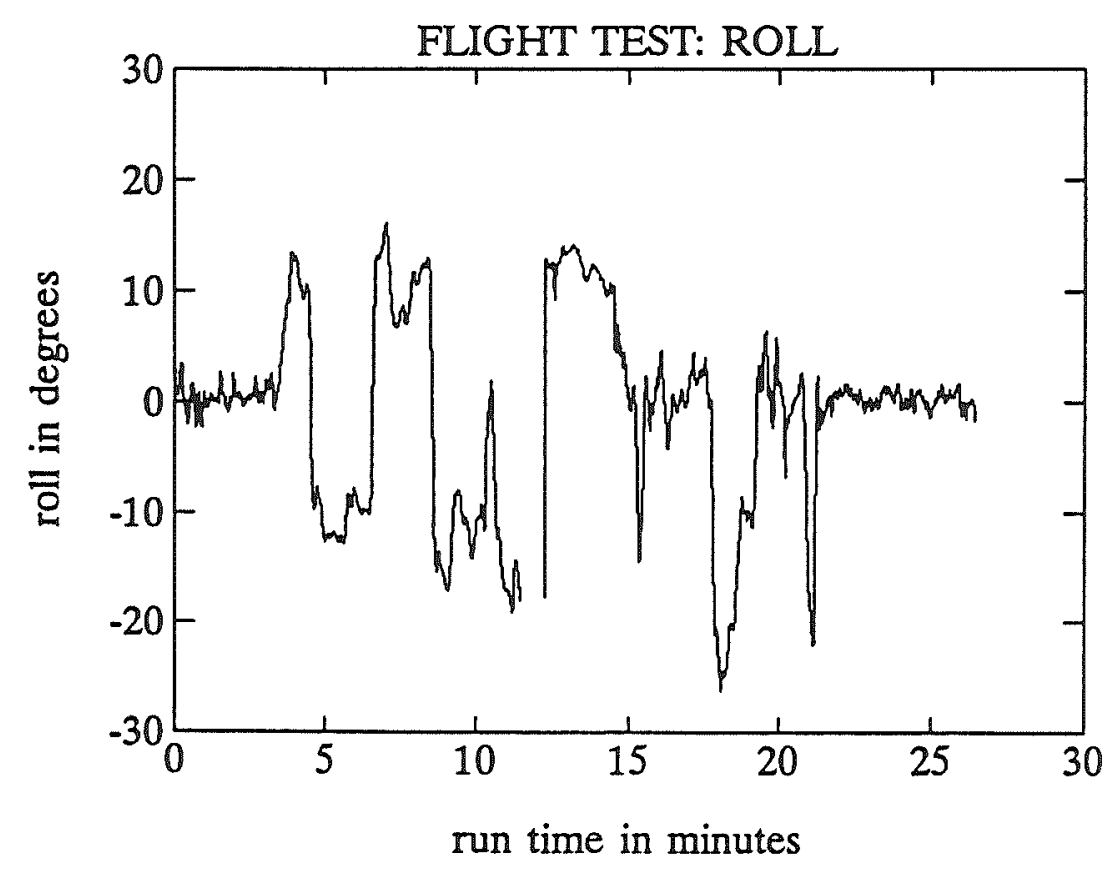

Figure 13. Flight test roll as a function of time.

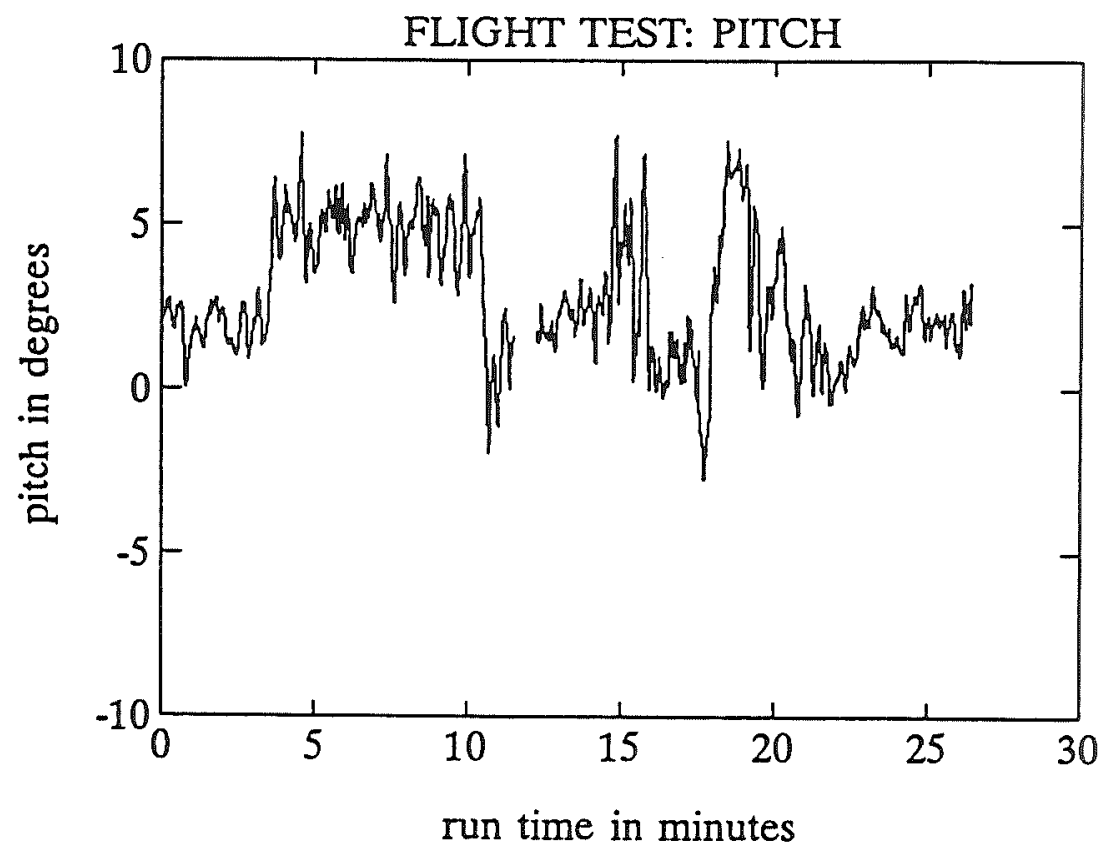

Figure 14. Flight test pitch as a function of time. 


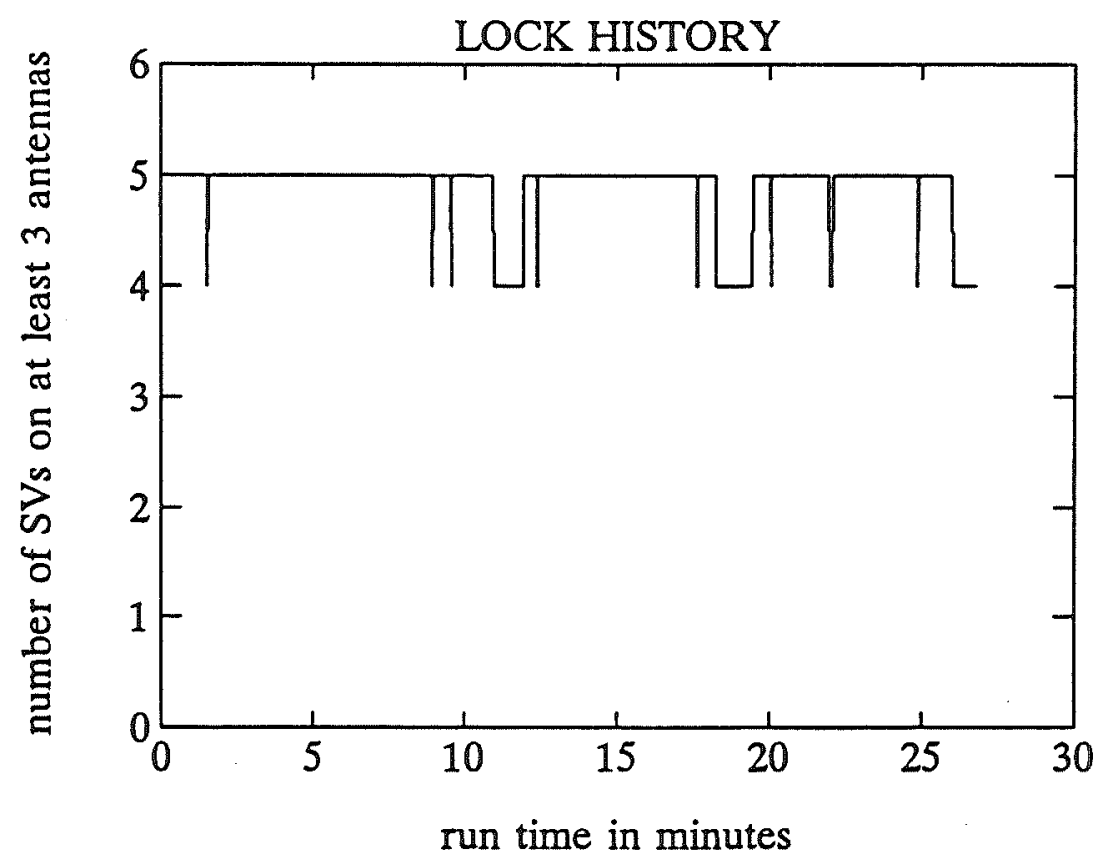

Figure 15. Flight test lock history.

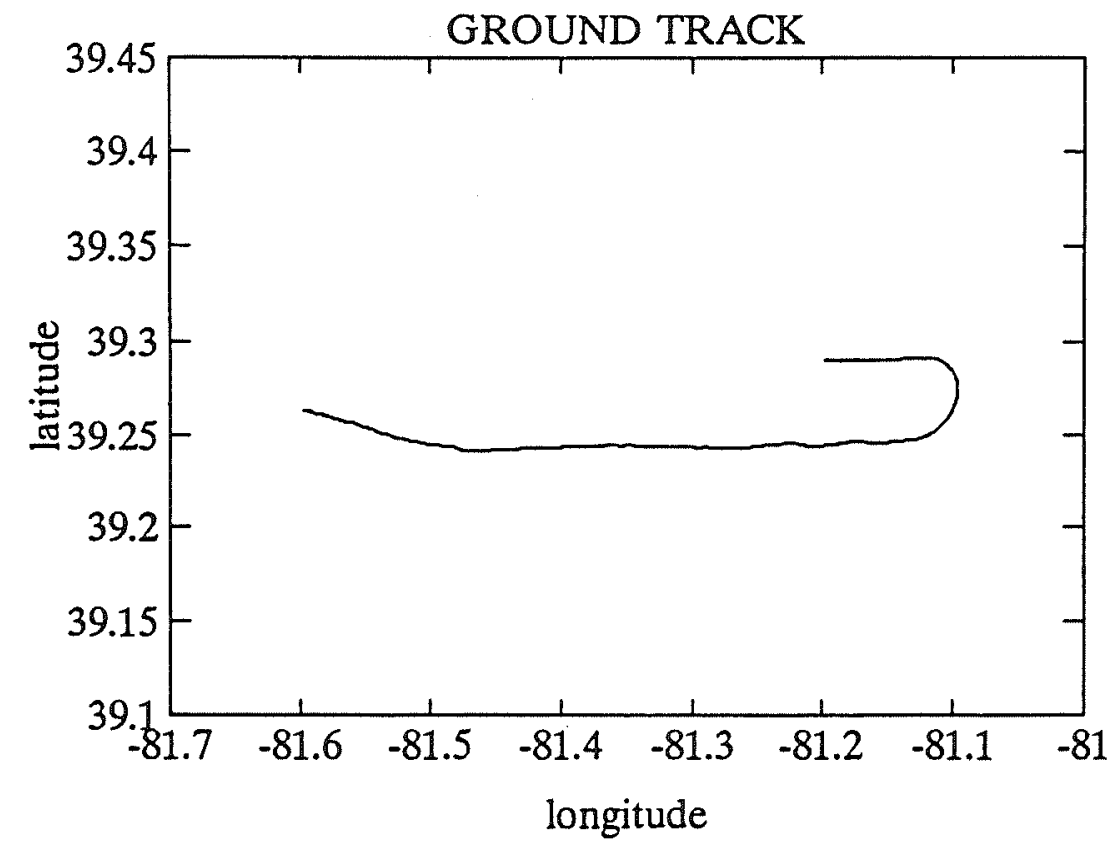

Figure 16. Ground track for a 13-minute section of the third flight test. 


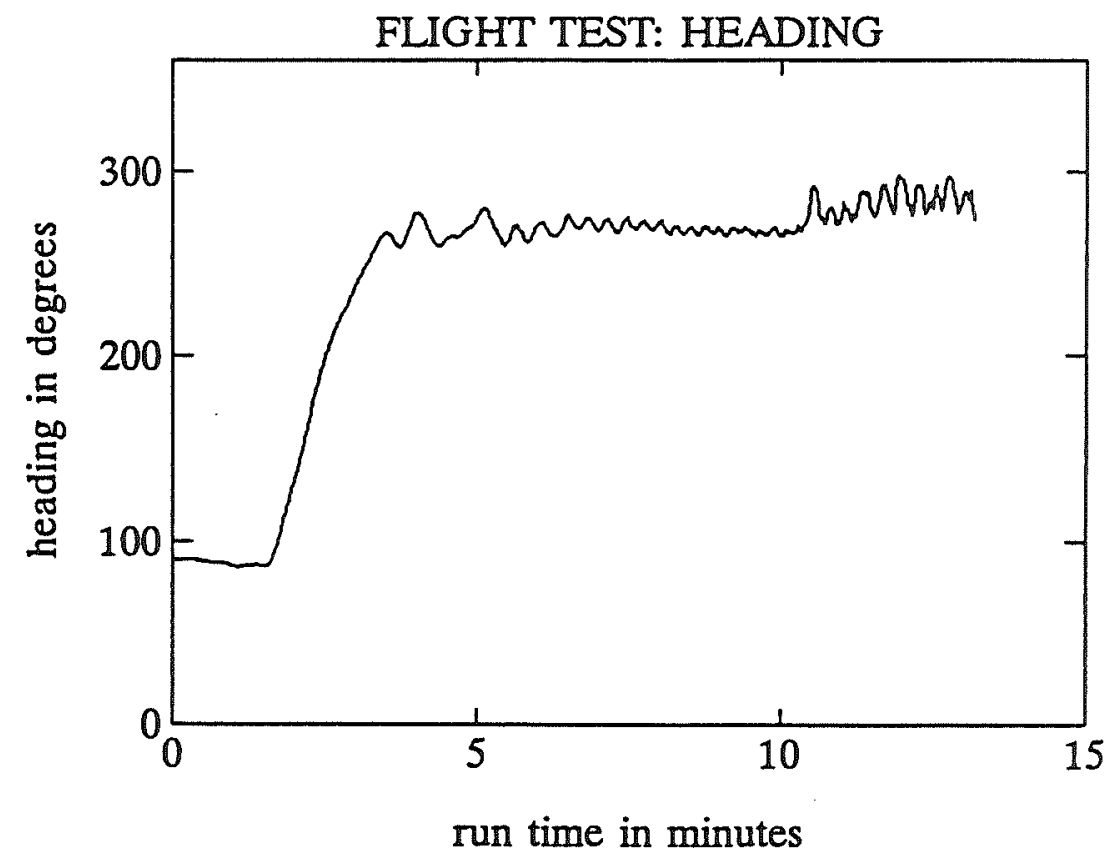

Figure 17. Flight test heading as a function of time.

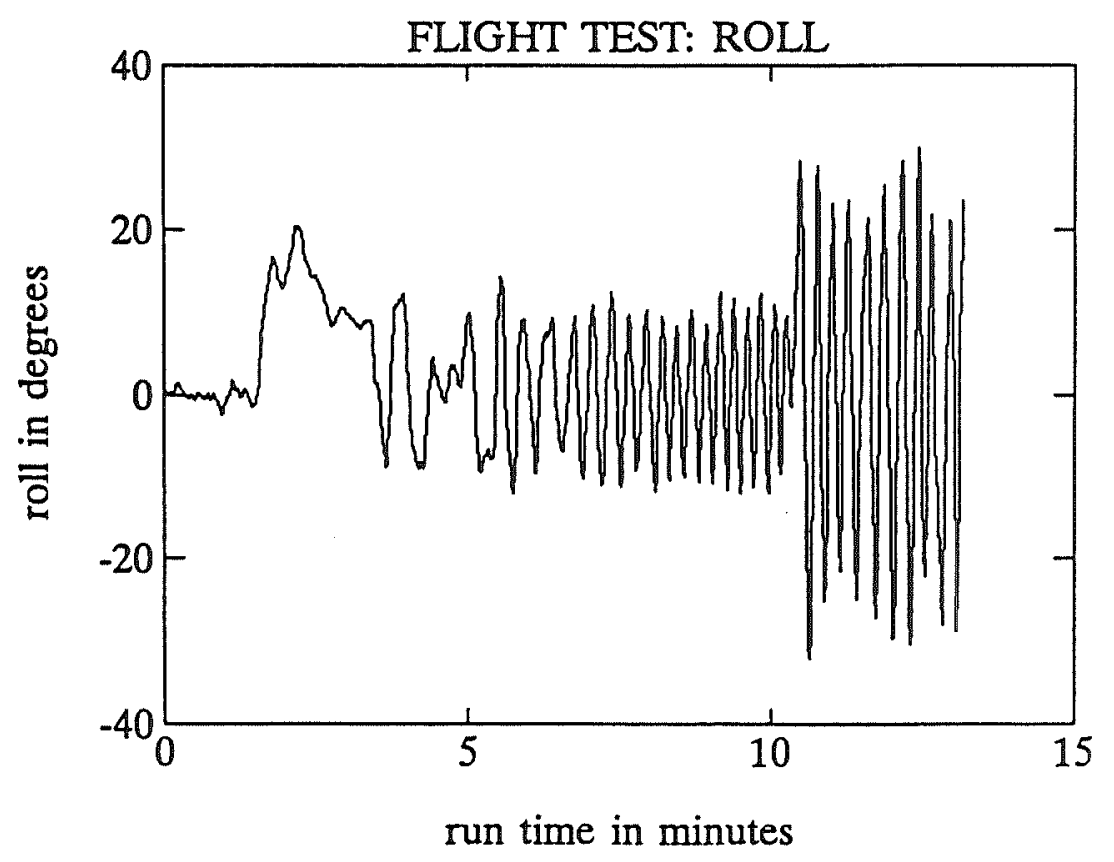

Figure 18. Flight test roll as a function of time. 


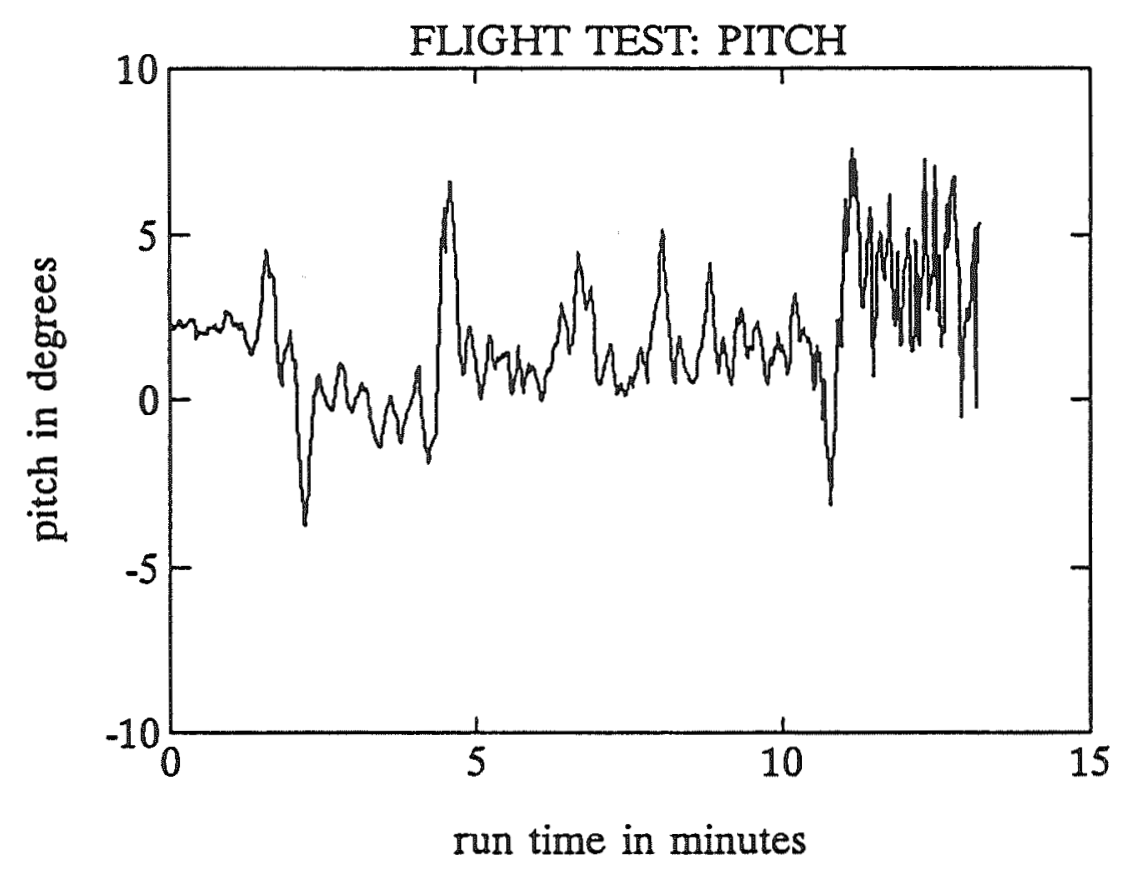

Figure 19. Flight test pitch as a function of time.

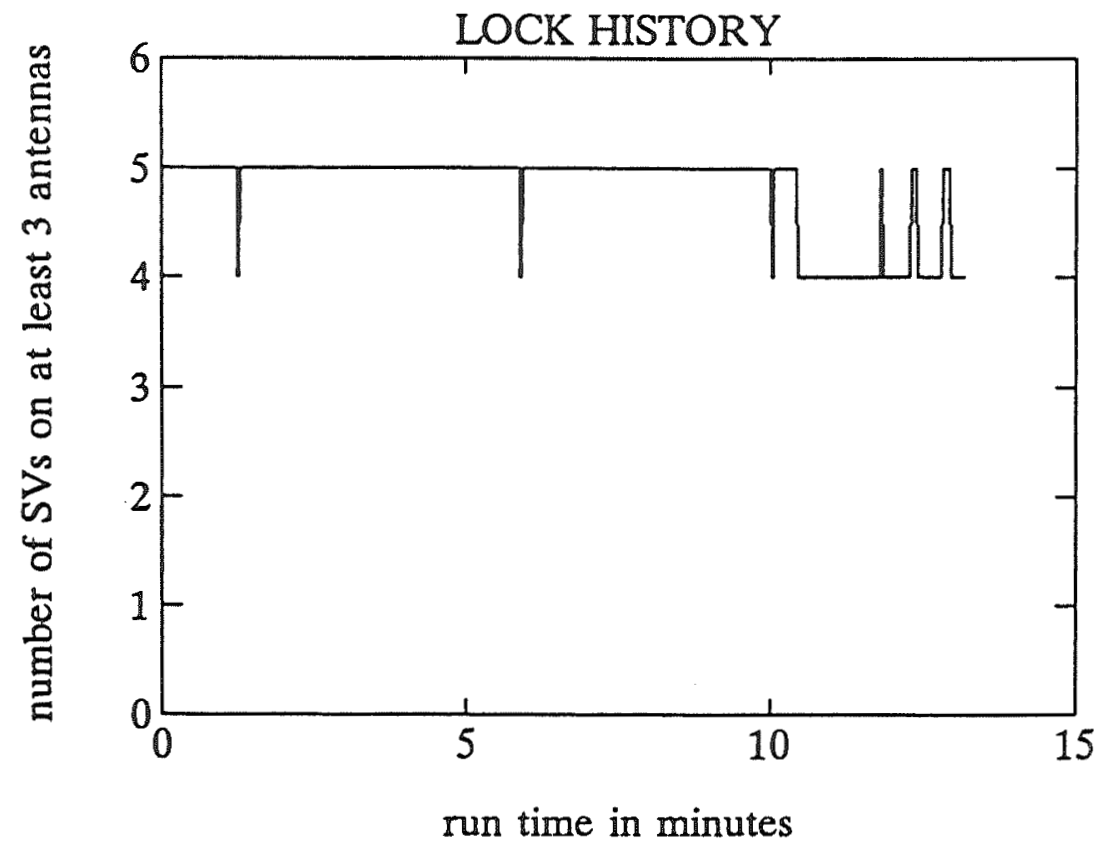

Figure 20. Flight test lock history. 